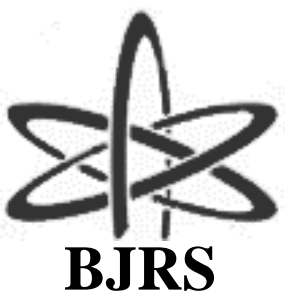

BRAZILIAN JOURNAL

$\mathrm{OF}$

RADIATION SCIENCES

02-3A (2014) 01-13

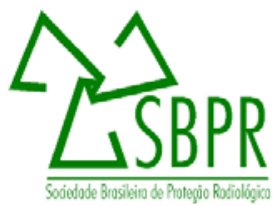

\title{
Análise de agrupamento na avaliação do comportamento de elementos quími- cos estáveis e parâmetros físico-químicos em efluente de mineração de urânio.
}

\author{
Wagner de S. Pereira ${ }^{1,2}$, Delcy de A.Py Júnior ${ }^{1}$,Luis A. de C. B. Dores ${ }^{1}$, Emanuele L. C. \\ Campelo $^{1}$, Gustavo F. de Morais ${ }^{1}$, Alphonse Kelecom ${ }^{1}$ e Juliana R. de S. Pereira ${ }^{3}$.
}

\author{
${ }^{1}$ Unidade de Tratamento de Minérios - UTM. \\ Caixa Postal 961, CEP 37.701-970, Poços de Caldas, MG, Brasil. \\ Indústrias Nucleares do Brasil - INB. \\ E-mail: wspereira@inb.gov.bre delcy@inb.gov.br
}

\begin{abstract}
${ }^{2}$ Laboratório de Radiobiologia e Radiometria Pedro Lopes dos Santos (LARARA-PLS), Grupo de Estudos em Temas Ambientais (GETA), Universidade Federal Fluminense UFF, C.P. 100436, CEP 24.001-970, Niterói, RJ, Brasil.

kelecom@uol.com.br ou wspereira@inb.gov.br
\end{abstract}

\begin{abstract}
${ }^{3}$ Aluna do Bacharelado interdisciplinar em Ciência e Tecnologia, Universidade Federal de Alfenas, Campus Poços de Caldas, Rodovia José Aurélio Vilela, 11999 - Poços de Caldas - MG, 37715-400

e-mail: pereirarsj@gmail.com
\end{abstract}

\begin{abstract}
RESUMO
A Unidade de Tratamento de Minério (UTM) é uma mina de urânio desativada. A análise estatística de agrupamento foi utilizada para avaliar o comportamento de elementos químicos estáveis e variáveis físico-químicas nos seus efluentes. A utilização da análise de agrupamento se mostrou eficiente na avaliação, permitindo identificar grupos de elementos químicos, de variáveis físico-químicas e de análises conjuntas (elemento e variáveis). Como resultado, podemos afirmar, com base na análise dos dados, uma forte ligação entre Ca e Mg e entre $\mathrm{Al}$ e $\mathrm{TR}_{2} \mathrm{O}_{3}$ (óxidos de terras raras) nos efluentes da UTM. O SO 4 também foi identificado como fortemente ligado aos sólidos totais e dissolvidos e estes ligados à condutividade elétrica. Outras associações existiram, mas não se mostraram tão fortemente ligadas. Coletas complementares para avaliação sazonal são requeridas para que as avaliações possam ser confirmadas. Análises estatísticas adicionais (técnicas de ordenação) devem ser utilizadas para tentar identificar as origens dos grupos identificados nessa análise.
\end{abstract}

\section{INTRODUÇÃO}

O Complexo Industrial de Poços de Caldas (CIPC) foi aprimeira unidade de mineração e processamento de urânio no Brasil. Ele está localizado no município de Caldas, planalto de Poços de Caldas, Minas Gerais, Brasil. Em 1996, com o fim da viabilidade econômica da exploração do 
urânio o CIPC foi desativado. Em 2004, o CIPC passou a ser denominado UTM (Unidade de Tratamento de Minério).

A mina a céu aberto, as pilhas de estéreis da mina (bota fora) e as barragens de rejeitos são fontes de elementosquímicos estáveis e radioativos, podendo causar impacto ambiental. Um programa de tratamento de efluentes foi criado com o objetivo de minimizar o impacto ambiental da UTM. Um programa de monitoramento ambiental foi mantido para confirmar a efetividade do tratamento de efluentes na mitigaçãodos impactos e na manutençãodos efluentes dentro dos limites permitidos pela legislação para a sua liberação para o ambiente.

Vários estudos foram realizados na região para avaliar o impacto radiológico ambiental (IRA) durante a operação da planta [1-4]. Outros autores focaram o transporte de radionuclídeos na região durante a mesma fase $[5,6]$. Antes da operação do CIPC, a linha de base da região foi estabelecida por Amaral e colaboradores [7].

A legislação brasileira sempre legislou sobre a dose adicional proveniente dos radionuclídeos [8, 9]. A gestão da pilha de estéril foi estudada por Fernandes [10] e Fernandes e colaboradores [11] visando o processo de descomissionamento da unidade.

A análise foi focada na liberação e dispersão de radionuclídeos para o meio ambiente. Atualmente um novo enfoque foi dado ao monitoramento ambiental, avaliando-se adicionalmente a liberação de elementosestáveis no meio ambiente.

Os elementosquímicos estáveis e radioativos podem causar vários problemas à saúde como, por exemplo, desordens neurotóxicas (Mn) ou disfunção renal (U) [12]. Para evitar esses efeitos, tornase necessárioomonitoramento das concentrações de elementos pesados em efluente, visando garantir que as concentrações dos mesmos estão dentro dos valores liberados pela legislação. A existência de drenagem ácida é a causa mais comum para contaminação da água por esses elementos no caso de minas. 
A drenagem ácida de minas é um problema comum e causa poluição ambiental [13, 14]. Na UTM, os estéreis são sulfetados e na presença de água, oxigênio e bactérias ferro e enxofre oxidantes produzem a drenagema ácida de mina (DAM). Este tipo de lixiviação passiva ocorre em outras minas, como em uma mina de urânio em Salamaca, Espanha onde também existem estas bactérias [15].

Visando utilizar a análise de agrupamento, método de análise estatística multivariada, na avaliação do comportamento dos elementosquímicos estáveis e de variáveis físico-químicas nos efluentes líquidosliberadosda UTM, foi realizada uma avaliação das variáveis físico-químicas (pH, turbidez, condutividade elétrica, potencial redox, oxigênio dissolvido, sólidos totais, suspensos e dissolvidos) e dos elementos químicos e compostos de elementos químicos estáveis (F, Na, K, Mo, Cr, Ni, $\mathrm{Cu}, \mathrm{Zn}, \mathrm{Cd}, \mathrm{V}, \mathrm{Ti}, \mathrm{Y}_{2} \mathrm{O}_{3}, \mathrm{Mg}, \mathrm{Al}, \mathrm{Si}, \mathrm{P}, \mathrm{Ca}, \mathrm{Mn}, \mathrm{Fe}, \mathrm{Ba}, \mathrm{SO}_{4}, \mathrm{TR}_{2} \mathrm{O}_{3}$ ) no efluente da UTM. Essa avaliação foi levada a termo com a aplicação da análise de agrupamento.

\section{MATERIAIS E MÉTODOS}

\subsection{Localização}

A UTM se localiza no município de Caldas, MG, Brasil. No seu interior encontra-se um divisor de águas de três corpos hídricos: Ribeirão das Antas, Ribeirão Soberbo e Córrego da Consulta. A UTM possuí três interfaces com o meio ambiente, uma em cada corpo hídrico. A interface da UTM no Ribeirão das Antas está localizada no ponto 014 (Fig. 1). Esse ponto é influenciado principalmente, pela cava da mina e pelo bota fora 8 .

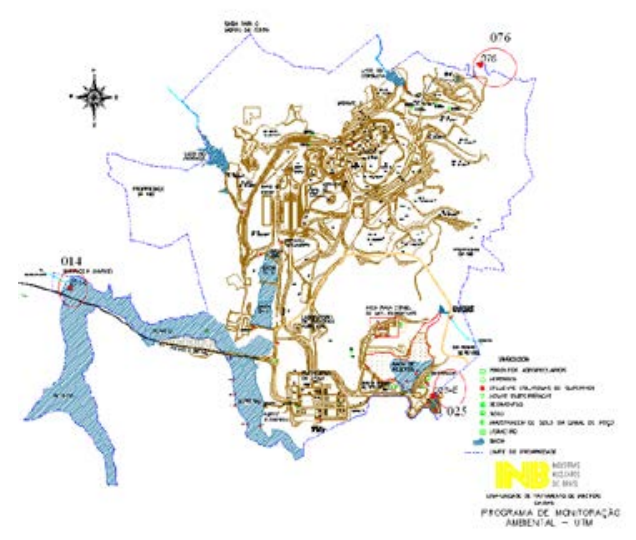

IX Latin American IRPA Regional Congress on Radiation Protection and Safety - IRPA 2013

Rio de Janeiro, RJ, Brazil, April 15-19, 2013

SOCIEDADE BRASILEIRA DE PROTEÇÃO RADIOLÓGICA - SBPR 
Figura 1 - Localização da área de estudo

No Ribeirão Soberbo a interface da UTM com omeio ambiente se encontra no ponto 025 (Fig.1) sendo influenciada pela bacia de rejeito e, por fim, a interface da UTM com o Córrego da Consulta está localizada no ponto 076(Fig. 1) e é influenciada pelo bota fora 4.

\subsection{Coletae Preparo de Amostras.}

As amostras foram coletadas nos meses de dezembro de 2009 a maio de 2010, perfazendo um total de 14 coletas. Alíquotas das amostras in natura foram utilizadas para a determinação da variáveis físico-químicas [16]. Frações das amostras foram aciduladas (1 $\mathrm{ml}$ de $\mathrm{HNO}_{3}$ por litro), filtradas em filtro de porosidade 0,45 $\mu \mathrm{m}$ [16]. A amostra filtrada foi utilizada para avaliação dos elementos químicos estáveis.

\subsection{Análise Química Dos Elementos Químicos Estáveis.}

O fluoreto foi analisado por eletrodo seletivo [17]. Análises dos elementose compostos Mo, Cr, $\mathrm{Ni}, \mathrm{Cu}, \mathrm{Zn}, \mathrm{Cd}, \mathrm{V}$, Ti, Y2O3, Mg, Al, Si, P, Ca, Mn, Fe, Ba, SO $\mathrm{TR}_{2} \mathrm{O}_{3}$ (óxidos de terras raras) foram realizadas por ICP OES[18]. Já os elementosNa e K foram analisados por fotômetro de chama. [19].

\subsection{Análise Físico-Químicas}

As análises de pH, foram realizadas seg. [20], as de turbiez (NTU) seg. [21]. As análises de condutividade elétrica ( $\mu \mathrm{S} \mathrm{cm}^{-1}$ ) seg.[22], as de potencial redox $(\mathrm{mV})$ seg. [23] e oxigênio dissolvido ( $\mathrm{mg} \mathrm{L}^{-1}$ ) seg. [24]. Os sólidos (totais, dissolvidos e em suspensão) em água foram analisados seg. [25].

\subsection{Análise Estatística De Agrupamento}

A análise de agrupamento visa agrupar objetos. O método consiste em reconhecer entre eles algum grau de semelhança que permita reuni-los em um mesmo grupo[26]. Nesse trabalho usaremos a análise de agrupamento (Cluster analysis, em inglês) no modo Ragrupando objetos pelos descritores[26] e [27]. 
Como este objeto utilizaremos as amostras coletadas e como descritores dos objetos os valores das variáveis físico-químicas e concentração dos elementos químicos estáveis.

Utilizamos como coeficiente de associação a relação de distância entre esses descritores. Utilizamos método de agrupamentoaglomerativo de Ward's (método pela variância mínima) que é modelado baseado nos processos de análise de variância [26], [27] e [28]. A idéia do método é aglomerar os grupos que minimizem soma dos quadrados dentro dos grupos, ou seja, a soma dos quadrados dos erros. Como coeficiente de associação a distância baseada no coeficiente r de Pearson (1-r de Pearson) [26], [27] e [28]. As análises foram realizadas no pacote estatístico Statistic, versão 7.3 .

\section{RESULTADOS E DISCUSSÃO}

\subsection{Análise De Agrupamento Dos Elementos Químicos Estáveis}

Os valores de concentração $\left(\mathrm{mgL}^{-1}\right)$ de, $\mathrm{Mo}, \mathrm{Cr}, \mathrm{Ni}, \mathrm{Cu}, \mathrm{Zn}, \mathrm{Cd}, \mathrm{V}, \mathrm{Ti}, \mathrm{Y}_{2} \mathrm{O}_{3}$ tiveram todos os valores abaixo do limite de detecção (LD), não podendo ser usados na análise. Já F, Na, K, Mg, $\mathrm{Al}, \mathrm{Si}, \mathrm{P}, \mathrm{Ca}, \mathrm{Mn}, \mathrm{Fe}, \mathrm{Ba}, \mathrm{SO}_{4}, \mathrm{TR}_{2} \mathrm{O}_{3}$, demonstraram valores de concentração acima do LD eforam utilizados na análise estatística. As concentrações dos elementos que ficaram acima do LD podem ser vistos na Tabela 1.

O resultado da análise de agrupamento pode ser vista na Figura 2. Nessa análise foram identificados três grupos de elementos. O primeiro com a menor distância de ligação média entre os três grupos identificados é formada por Ca, Mg e esses associados ao F. O segundo grupo foi composto por $\mathrm{SO}_{4}$ e K unidos a Mn e $\mathrm{P}$ e ambos unidos ao Na. O terceiro grupo, com maiores distâncias de ligação médias entre os três grupos identificados, é composto de $\mathrm{TR}_{2} \mathrm{O}_{3}$ e $\mathrm{Al}$, unidos ao Si e esses unidos aos $\mathrm{Ba}$ e Fe. As duas menores ligações foram entre Ca e $\mathrm{Mg}$ e entre $\mathrm{TR}_{2} \mathrm{O}_{3}$ e $\mathrm{Al}$.

Tabela 1 -Valores de concentração $\left(\mathrm{mg} \mathrm{L}^{-1}\right)$ dos elementos analisados com valores superiores ao LD.

$\begin{array}{lllllllllllllll}\text { Data } & \text { pH } & \text { F } & \text { Na } & \text { K } & \text { Mg } & \text { Al } & \text { Si } & \text { P } & \text { Ca } & \text { Mn } & \text { Fe } & \text { Ba } & \mathrm{SO}_{4} & \mathrm{TR}_{2} \mathrm{O}_{3}\end{array}$


$\mathrm{mg} \mathrm{L}^{-1}$

$\begin{array}{lllllllllllllll}10 / 12 / 09 & 6,24 & 0,96 & 0,8 & 0,8 & 0,38 & 0,11 & 1,76 & 0,05 & 12,7 & 0,54 & 0,05 & 0,05 & 29,3 & 0,50 \\ 07 / 01 / 10 & 6,12 & 1,81 & 0,8 & 2,3 & 1,73 & 0,05 & 1,68 & 0,05 & 79,3 & 1,28 & 0,44 & 0,07 & 38,8 & 0,65 \\ 14 / 01 / 10 & 6,64 & 1,30 & 1,1 & 2,8 & 0,56 & 0,09 & 2,04 & 0,05 & 16,5 & 1,00 & 0,69 & 0,68 & 34,8 & 0,65 \\ 20 / 01 / 10 & 6,76 & 1,05 & 0,8 & 2,5 & 0,47 & 0,24 & 1,97 & 0,05 & 13,3 & 0,05 & 3,76 & 0,06 & 30,8 & 0,65 \\ 27 / 01 / 10 & 6,65 & 1,30 & 1,0 & 2,5 & 0,51 & 0,05 & 2,20 & 0,05 & 20,3 & 0,70 & 0,15 & 0,08 & 54,2 & 0,65 \\ 09 / 02 / 10 & 7,65 & 1,22 & 1,1 & 2,5 & 0,45 & 0,48 & 1,93 & 0,05 & 12,8 & 0,97 & 0,09 & 0,05 & 36,1 & 0,73 \\ 23 / 02 / 10 & 6,67 & 0,91 & 1,0 & 2,7 & 0,55 & 1,59 & 2,47 & 0,06 & 20,1 & 1,34 & 1,13 & 0,05 & 47,1 & 1,15 \\ 03 / 03 / 10 & 7,05 & 1,35 & 0,8 & 3,1 & 0,48 & 0,08 & 2,13 & 0,05 & 24,8 & 1,28 & 0,05 & 0,06 & 56,6 & 0,65 \\ 09 / 03 / 10 & 6,57 & 1,21 & 0,8 & 3,1 & 0,50 & 0,07 & 2,06 & 0,08 & 28,0 & 1,21 & 0,05 & 0,05 & 64,1 & 0,78 \\ 23 / 03 / 10 & 6,77 & 2,10 & 0,8 & 3,0 & 0,64 & 0,05 & 2,42 & 0,05 & 31,7 & 0,76 & 0,05 & 0,05 & 63,9 & 0,65 \\ 07 / 04 / 10 & 6,53 & 1,15 & 0,7 & 2,9 & 0,49 & 0,06 & 2,03 & 0,05 & 26,6 & 0,84 & 0,05 & 0,05 & 62,2 & 0,65 \\ 13 / 04 / 10 & 6,81 & 1,66 & 1,8 & 2,6 & 0,49 & 0,10 & 2,21 & 0,06 & 27,7 & 0,79 & 0,05 & 0,05 & 58,4 & 0,70 \\ 20 / 04 / 10 & 6,60 & 1,45 & 1,3 & 2,3 & 0,49 & 0,05 & 1,91 & 0,05 & 30,7 & 0,82 & 0,22 & 0,05 & 73,8 & 0,70 \\ 27 / 04 / 10 & 6,70 & 1,70 & 1,4 & 2,8 & 0,56 & 0,10 & 1,69 & 0,05 & 41,1 & 1,06 & 0,05 & 0,05 & 95,3 & 0,70 \\ 04 / 05 / 10 & 6,84 & 1,22 & 0,9 & 2,7 & 0,53 & 0,05 & 1,86 & 0,06 & 31,6 & 0,87 & 0,05 & 0,05 & 77,2 & 0,70\end{array}$

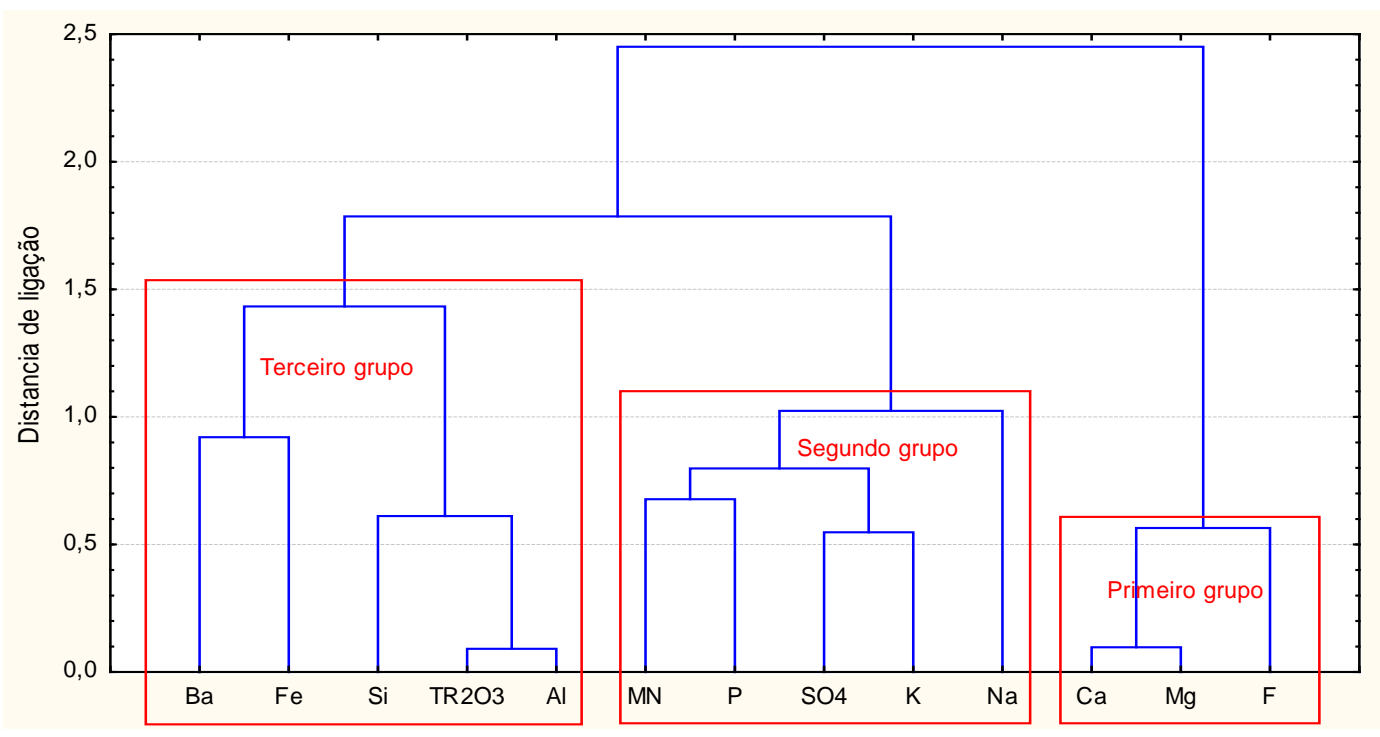

Figura 2 - Dendrogramados elementosanalisados, agrupados pelo método de Ward's e distâncias entre pontos medidas por 1-r de Pearson. 


\subsection{Análise De Agrupamento Das Variáveis Físico-Químicas}

Os dados das variáveis físico-químicas $\mathrm{pH}$, turbiez (NTU),condutividade elétrica $\left(\mu \mathrm{S} \mathrm{cm}^{-1}\right)$, potencial redox $(\mathrm{mV})$ e oxigênio dissolvido $\left(\mathrm{mg} \mathrm{L}^{-1}\right)$. Além de sólidos totais $\left(\mathrm{mg} \mathrm{L}^{-1}\right)$, suspensos (mg $\mathrm{L}^{-1}$ ) e dissolvidos $\left(\mathrm{mg} \mathrm{L}^{-1}\right)$. Podem ser vistos na Tabela 2.

A análise de agrupamento para as variáveis físico-químicas pode ser visualizada na Fig. 3. Essa análise identificou três grupos de variáveis. O primeiro grupo com sólidos totais e dissolvidos fortemente ligados e essas duas variáveis ligadas à condutividade elétrica. Os sólidos em suspensão ficaram ligados ao pH no segundo grupo. O terceiro grupo agrupou oxigênio dissolvido, potencial redox e turbidez.

Tabela 2 -Valores das variáveis físico-químicas analisadas.

\begin{tabular}{|c|c|c|c|c|c|c|c|c|}
\hline \multirow{3}{*}{ Data } & \multirow{3}{*}{$\mathbf{p H}$} & \multirow{2}{*}{ Turbidez } & \multirow{2}{*}{ Condut } & \multirow{2}{*}{ Pot Redox } & \multirow{2}{*}{ OD } & \multicolumn{3}{|c|}{ Sólidos } \\
\hline & & & & & & Totais & Suspensos & Dissolvidos \\
\hline & & NTU & $\mu \mathrm{Scm}^{-1}$ & $\mathrm{mV}$ & $\mathrm{mg} \mathrm{L}^{-1}$ & & $\mathrm{mg} \mathrm{L}^{-1}$ & \\
\hline $10 / 12 / 09$ & 6,24 & 2,0 & 144,7 & 302,5 & 6,46 & 67,5 & 1,5 & 66,0 \\
\hline 07/01/09 & 6,12 & 4,4 & 122,8 & 291,7 & 7,03 & 74,5 & 15,5 & 59,0 \\
\hline $14 / 01 / 10$ & 6,64 & 6,5 & 101,6 & 335,9 & 6,13 & 67,5 & 14,5 & 53,0 \\
\hline $20 / 01 / 10$ & 6,76 & 5,3 & 89,0 & 329,8 & 6,68 & 71,0 & 16,5 & 54,5 \\
\hline $27 / 01 / 10$ & 6,65 & 8,0 & 117,1 & 315,9 & 8,06 & 105,0 & 15,0 & 90,0 \\
\hline 09/02/10 & 7,65 & 5,5 & 112,3 & 337,1 & 6,04 & 93,0 & 15,0 & 78,0 \\
\hline $23 / 02 / 10$ & 6,67 & 4,6 & 135,1 & 290,7 & 6,52 & 99,0 & 3,0 & 96,0 \\
\hline 03/03/10 & 7,05 & 3,5 & 162,6 & 222,9 & 6,00 & 129,5 & 10,0 & 119,5 \\
\hline 09/03/10 & 6,57 & 1,8 & 227,0 & 329,7 & 6,53 & 119,5 & 2,0 & 117,5 \\
\hline $23 / 03 / 10$ & 6,77 & 4,3 & 173,1 & 478,0 & 6,80 & 115,5 & 1,5 & 114,0 \\
\hline 07/04/10 & 6,53 & 18,0 & 153,5 & 339,8 & 6,21 & 126,5 & 10,0 & 116,5 \\
\hline $13 / 04 / 10$ & 6,81 & 4,2 & 182,0 & 300,8 & 7,11 & 142,0 & 9,0 & 133,0 \\
\hline $20 / 04 / 10$ & 6,60 & 3,1 & 195,6 & 316,9 & 6,71 & 134,0 & 6,0 & 128,0 \\
\hline $27 / 04 / 10$ & 6,70 & 2,2 & 290,0 & 298,2 & 6,74 & 192,5 & 5,0 & 187,5 \\
\hline 04/05/10 & 6,84 & 2,5 & 219,0 & 279,3 & 6,52 & 152,5 & 1,0 & 152,5 \\
\hline
\end{tabular}

IX Latin American IRPA Regional Congress on Radiation Protection and Safety - IRPA 2013 


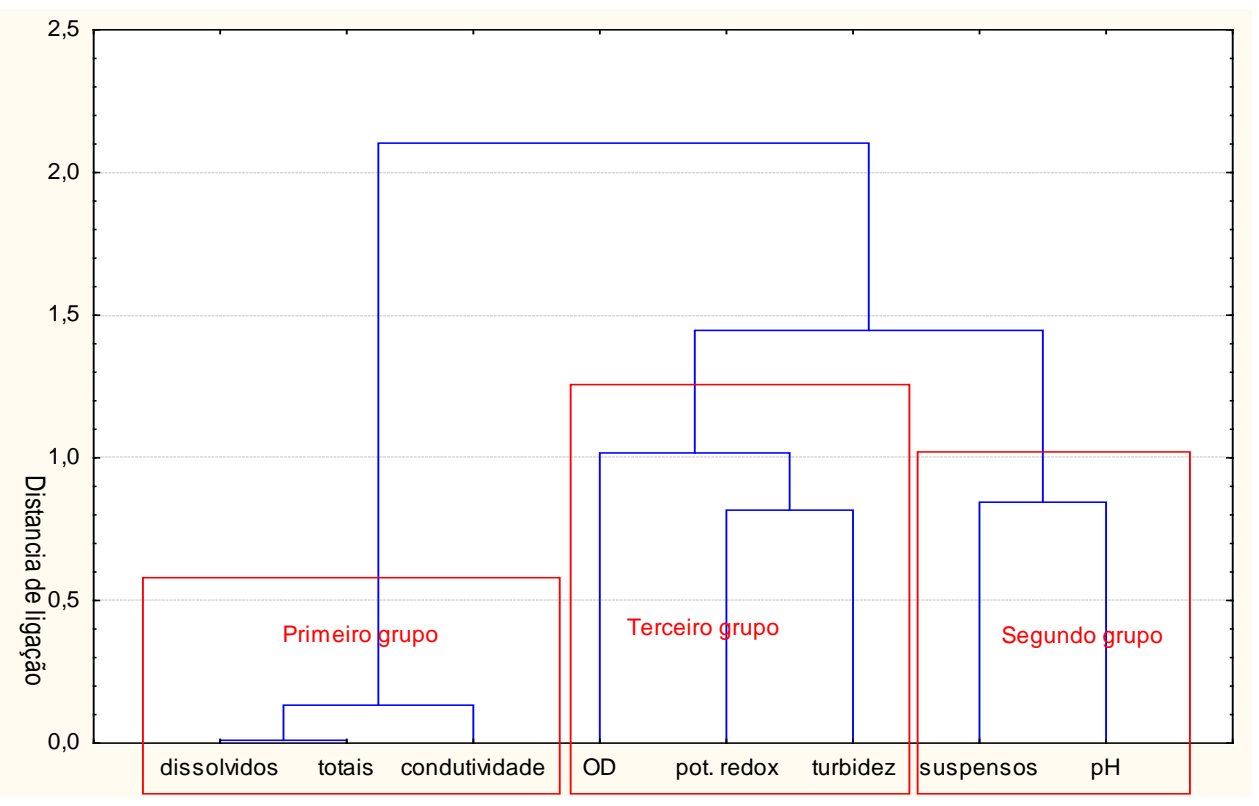

Figura 3 - Dendrograma das variáveis físico-químicas analisadas, agrupados pelo método de Ward's e distância entre pontos medidas 1-r de Pearson.

\subsection{Análise De Agrupamento Dos Parâmetros Físico-Químicos E Elementos Químicos Es- táveis.}

Avaliando-se os elementosquímicos estáveis e variáveis ambientais conjuntamente,a análise de agrupamento mostra ligações entre essas variáveis e elementos. $\mathrm{O} \mathrm{SO}_{4}$ está extremamente ligado aos sólidos dissolvidos e totais e também ligado à condutividade da água, como pode ser visto no grupo um da Fig. 4. O Ba e o Fe são ligados entre si e esses dois elementos ligados ao potencial redox e à turbidez do meio, como pode ser visto no grupo quatro da Fig.4.

Do grupo três fazem parte o Si e o K ligados ao pH;o P e o Mn ligados e esse sub-grupo ligados ao $\mathrm{Al}$ e $\mathrm{TR}_{2} \mathrm{O}_{3}$ que estão fortemente ligados. Esse últimosub-grupo (Mn, $\mathrm{P}, \mathrm{TR}_{2} \mathrm{O}_{3}$ e $\mathrm{Al}$ ) está ligado ao sub-grupo composto por Si, K e pH. Por fim o OD está ligado ao Na e esses aos sólidos em suspensão. O Ca e o Mg estão extremamente ligados e ambos ligados ao F, formando um subgrupo que se une ao sólido em suspensão, Na e OD. 


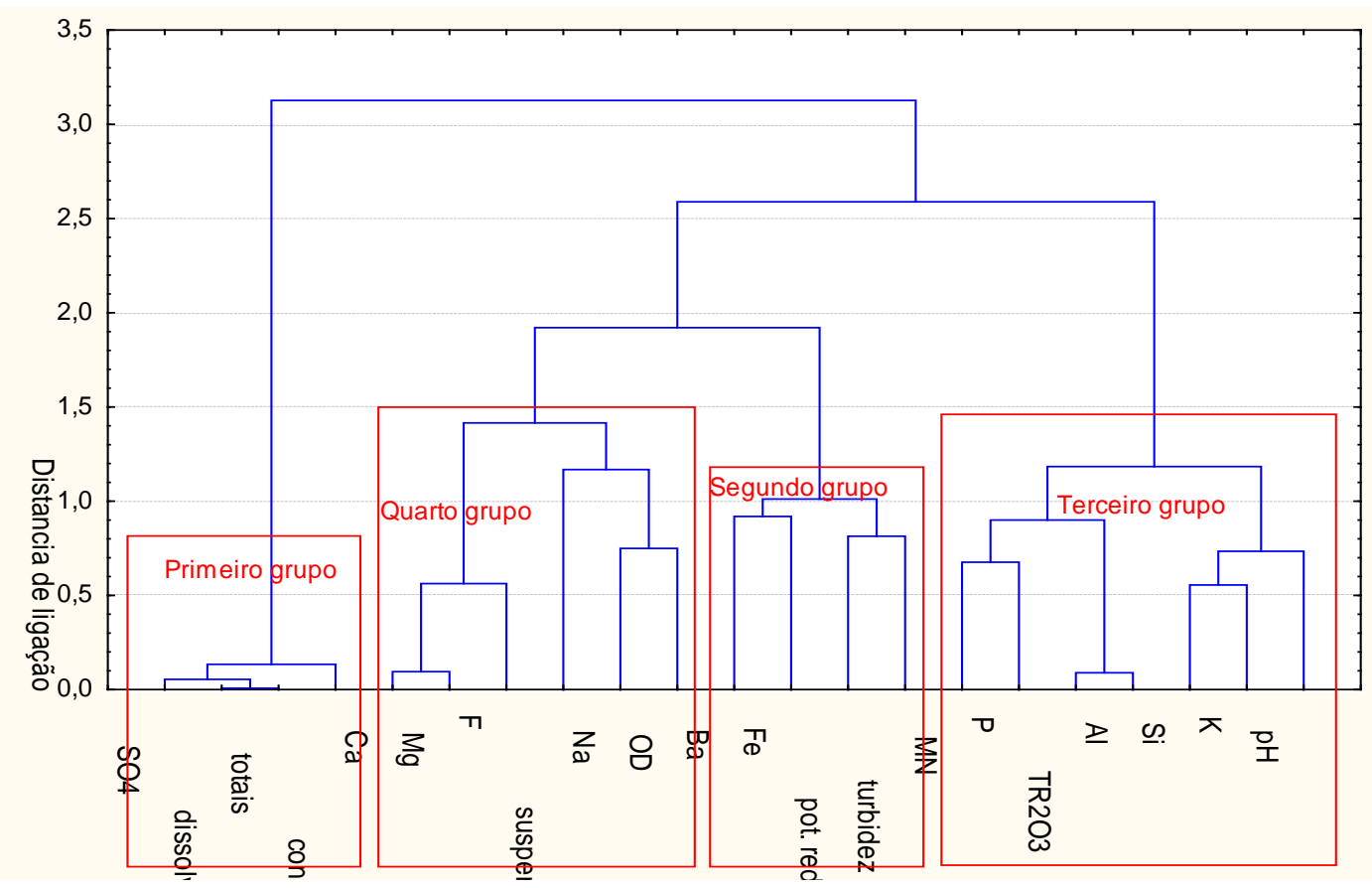

Figura 4 - Dendrograma dos elementos e das variáveis físico-químicas analisadas, agrupados pelo método de Ward's e distâncias entre pontos medidas por 1-r de Pearson.

\section{CONCLUSÃO}

A análise de agrupamento se mostrou uma ferramenta eficiente na avaliação da associação entre os elementosquímicos estudados e entre as variáveis físico-químicas estudadas e na avaliação dos elementos e variáveis conjuntamente.

A análise em separado (elementos químicos e variáveis físico-químicas) seguida da análise em conjunto, se mostrou importante, pois apontam associações distintas. Analisando-se as variáveis físico-químicas, observou-se a associação entre pH e sólidos em suspensão. Com a introdução dos elementos químicos na análise de agrupamento, esta associação foi desfeita, sendo o pH associado ao Si e K e os sólidos em suspensão ligados ao Na e oxigênio dissolvido. Os Si e K associados na análise conjunta demostram pouca associação quando a análise é feita só com os metais. 
O sulfato se mostrou fortemente ligado aos sólidos totais e dissolvido. Já os sólidos em suspensão se mostraram ligados ao Na e ao OD. O Ca e o Mg, se mostraram fortemente ligados, assim como os $\mathrm{TR}_{2} \mathrm{O}_{3}$ e Al.

A principal fonte de elementos químicos estáveis nos efluentes do ponto 014é a drenagem ácida no bota fora 4, cava da mina e área do entorno. O tratamento dos efluentes reduzem as concentrações dos elementos. A drenagem ácida é dependente de três fatores principais: água, pirita no minério e o oxigênio.

Variações sazonais nas concentrações de metais são esperadas, em função da disponibilidade de água e oxigênio nas pilhas que são função das chuvas na regiãoe do potencial de armazenagem de água entre seus grânulos de solo. Essa disponibilidade (de oxigênio e água) gera flutuações na produção de drenagem ácida e nas características dessa drenagem, alterando as características do termo fonte de metais.

As coletas foram realizadas na época do ano onde ocorrem os maiores índices de pluviometricidade.Análises em diferentes épocas do ano podem mostrar comportamentos nos elementos e variáveis diferentes.

Análises de ordenação outra técnica de análise multivariada, devem ser aplicadas visando explicar as associações identificadas na análise de agrupamento. Dentre as análises de ordenação a análise de componente principal, análise fatorial de correspondência ou análise de discriminante, podem ser aplicadas.

Esta avaliação deve ser considerada preliminar pelo baixo número de amostras analisadas e pela coleta realizada apenas na época chuvosa.

\section{REFERÊNCIAS}


[1] Fernandes, H.M., Veiga, L.H.S., Franklin, M.R., Prado, V.C.S. and Taddei, J.F. Environmental impact assessment of uranium mining and milling facilities: a study case at the Poços de Caldas uranium mining and milling site, Brazil. JournalofGeochemicalExploration, 52, pp. 161-173, (1994).

[2] Amaral, E.C.S. Modificação da exposição radiação natural devido a atividades agricolas e industriais numa área de radioatividade natural elevada no Brasil. Ph.D. thesis. Instituto de Biofisica, UFRJ, 130 pp. (in Portuguese). (1992).

[3] Amaral, E.C.S., Godoy, J.M., Rochedo, E.R.R., Vasconcellos, L.M.H. and Pires do Rio, M.A. The environmental impact of the uranium industry: Is the waste rock a significant contributor. Radiation Protection Dosimetry, 22, pp. 165-171. (1988).

[4] Amaral, E.C.S., Rochedo, E.R.R., Paretzke, H.G. and Penna Franca, E. The radiological impact of the agricultural activities in an area of high natural radioactivity.Radiation Protection Dosimetry, 45, pp 289-292. (1993).

[5] Azevedo, H. L. P., Amaral, E. C. S. and Godoy, J. M. Evaluation of the ${ }^{226}$ Ra transport by river sediments surrounding the brazilian uranium mining and milling facilities. Environmental Pollution, 51, pp 259-268. (1988).

[6] Barcellos, C., Amaral, E. \&Rochedo, E. Radionuclidetransportby Pocos de Caldas Plateaurivers, Brazil. Environ. Technol., 11, pp 533-40. (1990).

[7] Amaral, E.C.S., Azevedo, H.L.P. and Mendonca, A.H. Pre-operational environment survey at the uranium mine and mill site, Poços de Caldas, Minas Gerais, Brazil. Science ofthe Total Environment42, pp 257-266. (1985).

[8] COMISSÃO NACIONAL DE ENERGIA NUCLEAR, Norma CNEN-NE-3.01, Diretrizes Básicas de Radioproteção, 121 pp. (1988).

[9] COMISSÃO NACIONAL DE ENERGIA NUCLEAR, Norma CNEN-NN-3.01, Diretrizes Básicas de Proteção Radiológica, 34 pp. (2005). 
[10] Fernandes, H.M., Franklin, M.R., Veiga, L.H.S., Freitas, P. and Gomiero, L.A. Management of Uranium Mill Tailings: Geochemical Processes and Radiological Risk Assessment. Journal of Environmental Radioactivity, 30(l), pp 69-95. (1996).

[11] Fernandes, H.M. Subsidios ao Descomissionamento da Primeira Indústria de mineração e beneficiamento de urânio no Brasil. O caso do Complexo Minero Industrial de Pocos de Caldas Niterói. D.Sc. thesis, Departamento de Geoquímica da Universidade Federal Fluminense. 250 pp (in Portuguese). (1997).

[12] Nordberg, G.F., Fowler, B. A., Nordberg, M., Friberg, L., Handbook on the toxicology of metals, Third Edition, p. 154.

[13] Johnson, D.B., Hallberg K.B., Acid Mine Drainage Remediation Options: AReview, Science of the Total Environment 338, pp 3-14. (2005).

[14] Akcil, A., Koldas, S., Acid Mine Drainage (AMD): causes, tratment and case studies, Journal of Cleaner Production14, pp 1139-1145. (2006).

[15] Mata, Y. N., González F., Ballester, A., Blásquez M. L., Muñoz J. A., Inhibition of acid rock drainage from uranium ore waste using a conventional neutralization and precipitation treatment, Minerals Engineering15, pp 1141-1150. (2002).

[16] INDÚSTRIAS NUCLEARES DO BRASIL, IA-UTM-MA-04, Coleta e preparo de amostras ambientais na Unidade de Tratamento de Minério, 8 PP, (2006a)

[17] INDÚSTRIAS NUCLEARES DO BRASIL, IA-UTM-CP-027, Determinação de fluoreto em águas - eletrodo seletivo, 7pp, (2007a).

[18] INDÚSTRIAS NUCLEARES DO BRASIL, IA-UTM-CP-038, Matriz "AGUA" - Análise por ICP, 7pp, (2006b)

[19] INDÚSTRIAS NUCLEARES DO BRASIL, IA-UTM-CP-033, Determinação de sódio e potássio por fotometria de chama, 8 pp (2004).

[20] INDÚSTRIAS NUCLEARES DO BRASIL, IA-UTM-CP-031, Determinação potenciométrica de pH em águas, 10 pp, (2007b) 
[21] INDÚSTRIAS NUCLEARES DO BRASIL, IA-UTM-CP-032, Determinação de turbidez em águas, 7 pp, (2007c)

[22] INDÚSTRIAS NUCLEARES DO BRASIL, IA-UTM-CP-041, Determinação de condutividade eletrica em águas, 8 pp, (2007d)

[23] INDÚSTRIAS NUCLEARES DO BRASIL, IA-UTM-CP-068, Determinação de potencial redox, 6 pp, (2008a).

[24] INDÚSTRIAS NUCLEARES DO BRASIL, IA-UTM-CP-069, Determinação de oxigênio dissolvido em água, 9 pp, (2008b).

[25] INDÚSTRIAS NUCLEARES DO BRASIL, IA-UTM-CP-052, Determinação de sólidos (totais, em suspensão e dissolvidos) em água, 9 pp, (2007e).

[26] Ferreira, D. F. “Estatística Multivariada”. (1 ed.). Lavras: editora da UFLA. (2008).

[27] Valentin, J. L. “Ecologia numérica. Uma introdução à análise multivariada de dados ecologicos”. Rio de Janeiro: Interciência. (2000).

[28] Gotelli, N. J., \& Ellison, A. M. (2004). A primer of ecological statistic.sunderland: Sinauer associates. $510 \mathrm{pp}$. 